\title{
High nuclear expression levels of histone-modifying enzymes LSD1, HDAC2 and SIRT1 in tumor cells correlate with decreased survival and increased relapse in breast cancer patients
}

Remco S Derr ${ }^{1 \dagger}$, Anneke Q van Hoesel ${ }^{1 \dagger}$, Anne Benard $^{1}$, Inès J Goossens-Beumer ${ }^{1}$, Anita Sajet ${ }^{1}$, N Geeske Dekker-Ensink1', Esther M de Kruijf ${ }^{1}$, Esther Bastiaannet ${ }^{1}$, Vincent THBM Smit ${ }^{2}$,

Cornelis JH van de Velde ${ }^{1}$ and Peter JK Kuppen ${ }^{1 *}$

\begin{abstract}
Background: Breast cancer is a heterogeneous disease with a highly variable clinical outcome in which both genetic and epigenetic changes have critical roles. We investigated tumor expression levels of histone-modifying enzymes LSD1, HDAC2 and SIRT1 in relation with patient survival and tumor relapse in a retrospective cohort of 460 breast cancer patients. Additionally, we correlated expression levels with tumor differentiation and tumor cell proliferation.
\end{abstract}

Methods: Immunohistochemical staining for LSD1, HDAC2 and SIRT1 was performed on tissue microarrays of tumor and corresponding normal formalin-fixed paraffin-embedded tissues from breast cancer patients. Median nuclear expression levels in tumor tissues were used to divide the patients into low and high expression categories. In combined expression analyses, patients were divided into four subgroups: 1, all enzymes below-median; 2, one enzyme above-median; 3, two enzymes above-median; 4, all three enzymes above-median. The Cox proportional hazard model was used for univariate and multivariate survival analyses. The Pearson Chi-square method was used to assess correlation of combined expression levels with tumor cell proliferation and tumor differentiation.

Results: Expression of LSD1 and SIRT1, but not of HDAC2, was significantly increased in tumor tissues compared to their normal counterparts (both $p<0.001$ ). Multivariate survival analyses identified SIRT1 as independent prognostic factor for relapse-free survival (RFS) with a hazard ratio (HR) of $1.34(95 \% \mathrm{Cl}=1.04-1.74, p=0.02)$. For overall survival $(\mathrm{OS})$, no significant differences were found when the individual enzymes were analyzed. Analyses of combined expression levels of the three histone-modifying enzymes correlated with OS $(p=0.03)$ and RFS $(p=0.006)$ with a HR of respectively $1.49(95 \% \mathrm{Cl}=1.07-2.08)$ and $1.68(95 \% \mathrm{Cl}=1.16-2.44)$ in multivariate analyses and were also related to tumor differentiation $(p<0.001)$ and tumor cell proliferation $(p=0.002)$.

Conclusions: When the combined expression levels were analyzed, high expression of LSD1, HDAC2 and SIRT1 showed shorter patient survival time and shorter time to tumor relapse and correlated with poor tumor differentiation and a high level of tumor cell proliferation. Expression of these histone-modifying enzymes might therefore be involved in breast cancer pathogenesis.

Keywords: Biomarkers, Breast cancer, Clinical outcome, Epigenetics, HDAC2, Histone-modifying enzymes, LSD1, SIRT1

\footnotetext{
* Correspondence: p.j.k.kuppen@lumc.nl

${ }^{\dagger}$ Equal contributors

'Department of Surgery, K6-R, Leiden University Medical Center (LUMC), P.O. Box 9600, 2300 RC Leiden, The Netherlands

Full list of author information is available at the end of the article
} 


\section{Background}

Clinical outcome of breast cancer patients is widely variable, due to the molecular heterogeneity of breast cancer. Breast cancer classification is based on a combination of several clinicopathological parameters, including histopathology, tumor stage, tumor grade and hormone receptor status and are used to guide treatment of breast cancer patients [1]. Even so, both over- and undertreatment of individual breast cancer patients occur, due to lack of reliable biomarkers [2,3]. In order to further subclassify breast cancer patients, new prognostic biomarkers are warranted to improve the prognosis of individual breast cancer patients, based on their tumor characteristics. Such molecular biomarkers can be derived from biological mechanisms that underlie tumor growth and development.

Epigenetics is a rapidly developing field of research. Epigenetic mechanisms include DNA methylation, histonemodifying enzymes and their histone modifications. Due to the reversible nature of these processes, they are attractive targets for drug development and could be exploited to find novel prognostic biomarkers [3]. Histone-modifying enzymes are responsible for modification of certain residues on histone tails (histone modifications), thereby regulating DNA accessibility and expression of specific genes. Aberrant expression of histone-modifying enzymes, including lysine-specific demethylase 1 (LSD1), histone deacetylase 2 (HDAC2) and silent mating-type information regulation 2 homologue 1 (SIRT1), has been shown to have a role in breast cancer development [4-9] as well as prognostic value for breast cancer [10]. LSD1 is the first identified histone demethylase involved in specific demethylation of mono- and dimethylated lysine 4 on histone 3 (H3K4) and lysine 9 on histone 3 (H3K9) [4], and has been shown to increase with tumor progression [5]. HDAC2 is part of the class I HDACs and is responsible for deacetylation of histones and other protein targets [6]. Deacetylation of histones leads to compaction of the chromatin (heterochromatin) and reduced transcription of genes, including genes involved in processes such as cellular proliferation and cellular differentiation [6]. HDAC inhibition is currently investigated in clinical trials aiming to reverse hormone resistance in breast cancer [7]. SIRT1 deacetylates several histones and plays a role in tumorigenesis [8] and expression levels were increased in breast tumors compared to their matched normal breast tissues [9]. Recently, two publications showed that both histone demethylation inhibitors and histone deacetylation inhibitors, and especially a combination of the two agents, inhibit breast cancer cell growth in vitro [11,12], suggesting an important role for histone demethylases and deacetylases in breast cancer.

LSD1, HDAC2 and SIRT1 are shown to act together in a single complex that represses transcription through compaction of the chromatin [13], thereby regulating gene expression. Therefore, we hypothesized that the combined expression levels of these collaborating histonemodifying enzymes in breast tumors is a stronger predictor for patient survival and tumor relapse than expression levels of the individual enzymes. Therefore, we investigated the correlation of the nuclear expression levels of LSD1, HDAC2 and SIRT1 as well as the combined expression levels of these enzymes with clinical outcome. The results showed that the expression levels of LSD1 and SIRT1 were increased in tumor tissues compared to adjacent normal breast tissues. Furthermore, overall survival (OS) and relapse-free survival (RFS) were decreased in breast cancer patients when tumor cells expressed high levels of all three markers. Finally, combined expression levels of the histone-modifying enzymes LSD1, HDAC2 and SIRT1 correlated with tumor differentiation and tumor cell proliferation.

\section{Methods}

\section{Patient selection}

The patient population was a retrospective cohort of female breast cancer patients (TNM: I-III) who underwent primary tumor resection at the Leiden University Medical Center (LUMC) between 1985 and $1996(\mathrm{n}=822)$, as described previously [14]. Patients with bilateral tumors or a prior history of cancer (other than basal cell carcinoma or cervical carcinoma in situ) were excluded from the study. The following data were retrieved and used as covariates in multivariate analyses: age, tumor size, nodal status, expression of estrogen receptor (ER), progesterone receptor (PgR), human epidermal growth factor 2 (HER2), tumor grade, histological type, local and systemic therapy, survival time, and time until tumor relapse. All tumors were graded and histologically classified according to pathological standards by an experienced breast cancer pathologist (V.S.). The study was conducted with anonymized patient data according to Dutch law and in agreement with the Dutch Code of Conduct: "Proper Secondary Use of Human Tissue in the Netherlands" (Federation of Medical Scientific Societies, the Netherlands, http:// www.federa.org/sites/default/files/bijlagen/coreon/code propersecondaryuseofhumantissue1_0.pdf). The specific section is paragraph one of chapter eight on page 43 and therefore we did not ask for approval of an ethics committee [15], and according to the REMARK guidelines [16].

\section{Study design}

Formalin-fixed paraffin-embedded (FFPE) tumor tissue of 701 patients, of whom tumor tissue was available, was included into a tissue microarray (TMA), as described previously [14]. For each patient, three cores of tumor tissue were included. For 261 breast cancer patients, of 
whom normal epithelial breast tissue was available, three cores of normal breast tissue were included in separate TMA blocks.

\section{Immunohistochemistry}

TMA sections were cut $(4 \mu \mathrm{m})$ and processed for immunohistochemistry (IHC). The antibodies that were used for IHC were validated by several other research groups: anti-LSD1 (ab17721, mouse, Abcam, Cambridge, United Kingdom) [17,18], anti-HDAC2 (ab39669, rabbit, Abcam) and anti-SIRT1 (ab32441, rabbit, Abcam) [19]. The IHC was performed using a standard protocol [20]. Briefly, tissues were deparaffinized in xylene and rehydrated in a series of graded alcohol. Antigen retrieval was performed by heating the sections for $10 \mathrm{~min}$ in sodium-citrate buffer at $95^{\circ} \mathrm{C}(\mathrm{pH} 6.0)$. Endogenous peroxidase activity was blocked with $0.3 \%$ hydrogen peroxide solution for $20 \mathrm{mi}-$ nutes. Incubation, with an optimized concentration of the antibodies described, was performed overnight at room temperature. Envision + peroxidase labelled polymer rabbit or mouse (Dako, Glostrup, Denmark) and DAB + liquid substrate chromogen system (Dako) were used for visualization of the expression levels. Counterstaining was performed using haematoxylin and dehydration was performed using graded alcohol and xylene.

\section{Evaluation of immunohistochemistry}

The scoring of the immunohistochemical staining was performed by two investigators (A.S. and G.D.), who were blinded for the clinicopathological data. The percentage of positive stained tumor cell nuclei was scored in each of the tissue cores, from $0-100 \%$ with $10 \%$ increments. The second observer scored $30 \%$ of the tissue cores in order to determine consistency in quantification, which was tested with Cohen's kappa coefficient for inter-observer variability. A Cohen's kappa coefficient $>0.6$ was considered as substantial agreement. In addition to tumor tissues, stained normal epithelial breast tissue cores were also evaluated using the same scoring criteria as described above.

\section{Statistical analysis}

Data were analyzed using SPSS 20.0 for Windows (SPSS Inc., Chicago, Illinois, United States of America). The paired student's t-test was used to compare expression levels in tumor breast tissues and their corresponding normal epithelial tissues of 60 individual patients. The one-way ANOVA method was used for calculation of differences in expression levels between the TNM tumor stages (I-III) for LSD1, HDAC2 and SIRT1. For survival analyses, the patients were divided into a low and high expression category based on the median percentage positive tumor cell nuclei per enzyme. The Cox proportional hazards model was used for univariate and multivariate survival
Table 1 Clinicopathological data of the $\mathbf{4 6 0}$ breast cancer patients used in the study

\begin{tabular}{llll}
\hline $\begin{array}{l}\text { Characteristic } \\
\text { Follow-up (years) }\end{array}$ & $\begin{array}{l}\text { Mean (range) } \\
\mathbf{1 1 . 8 ( 0 . 1 6 - 2 7 . 5 5 )}\end{array}$ & $\mathbf{N}=\mathbf{4 6 0}$ & $\%$ \\
\hline Age (years) & $58.3(23-89)$ & & \\
$<45$ & & 90 & 20 \\
$45-55$ & 106 & 23 \\
$55-65$ & 107 & 23 \\
$\geq 65$ & 157 & 3 \\
Tumor size (T) & & \\
1 & & 182 & 40 \\
2 & & 227 & 49 \\
$3-4$ & 51 & 11
\end{tabular}

Nodal status (N)

Negative

$236 \quad 51$

Positive

ER

Positive

PgR

Positive

HER2

No overexpression

Overexpression

Histologic type

Ductal

Other

Tumor grade

1

2

3

Local treatment

Mastectomy without RT $\quad 180 \quad 39$

$\begin{array}{lll}\text { Mastectomy with RT } & 97 & 21\end{array}$

BCS with RT $183 \quad 40$

Systemic treatment

Chemotherapy alone $\quad 89 \quad 19$

$\begin{array}{lll}\text { Endocrine therapy alone } & 77 & 17\end{array}$

Chemo- and endocrine therapy $\quad 18 \quad 4$

None $\quad 276 \quad 60$

Clinicopathological characteristics of the cohort of breast cancer patients. Statistical analyses were performed with all patients $(n=460)$ with complete clinicopathological data and nuclear staining data for LSD1, HDAC2 and SIRT1. Tumor size (T) and nodal status (N) were based on the TNM staging criteria. ER: estrogen receptor, PgR: progesterone receptor, HER2: human epidermal growth factor receptor 2, RT: radiotherapy, BCS: breast conserving surgery. 
analyses. Kaplan-Meier (KM) curves and cumulative incidence curves were plotted to graphically show differences in patient survival and tumor relapse between the groups with different expression levels, respectively. For the uniand multivariate analyses, only patients with nuclear staining data for all three enzymes and all covariates available, complete case analysis, were used in the statistical analyses $(n=460)$. Data were censored when patients were alive or free of relapse at their last follow-up date (lastly march 2013). Overall survival (OS) was defined as the time from date of surgery until death from any cause. Relapse-free survival (RFS) was defined as the time from surgery until the occurrence of a local, regional or distant tumor relapse or death by cancer. The Pearson Chi-square method was used to test for correlations between the combined expression levels of LSD1, HDAC2 and SIRT1 and clinical parameters. The low expression group was used as a reference in the single marker analyses. Low expression of all three markers was used as reference in the analyses of the combined expression levels. For the analyses of the combined expression levels of the markers, the patients were divided into four categories as follows: all enzymes belowmedian expression ('all-low'), one enzyme above-median expression, two enzymes above-median expression and all three enzymes above-median expression ('all-high'). We performed a Chi-square test between the four patients groups and all variables used as covariates, which are wellknown independent prognostic factors in breast cancer and we corrected for those covariates in the multivariate analyses. For all analyses, a two-sided p-value $\leq 0.05$ was considered statistically significant.

\section{Results}

Immunohistochemical staining of LSD1, HDAC2 and SIRT1 in breast tumors

Table 1 shows the clinicopathological data of the breast cancer patients $(n=460)$ used for the statistical analyses of the three markers. The mean follow-up time was 11.8 years (range: $0.16-27.55$ years) and the mean age at diagnosis was 58.3 years (range: $23-89$ years). Percentages of positive nuclei for LSD1, HDAC2 and SIRT1 in the tumor and normal tissue cores were determined by IHC. Figure 1 shows representative pictures of normal breast tissue cores immunohistochemically stained individually for each enzyme, as well as representative pictures of breast cancer tissue cores with expression above and below median for each of the enzymes. The brown color is the amount of expression of the enzyme. The median percentages of positive tumor nuclei, used for the statistical analyses, were $85 \%$ for LSD1, $80 \%$ for HDAC2 and 70\% for SIRT1. Cohen's kappa coefficient was calculated to determine the inter-observer variability. The kappa coefficients for scoring of the tumor tissues were 0.664 for LSD1 and 0.627 for SIRT1. Both kappa coefficients were considered as substantial agreement between the observers. For staining of HDAC2 in tumor tissues, the kappa for scoring of the tumor tissue was not considered as substantial agreement.

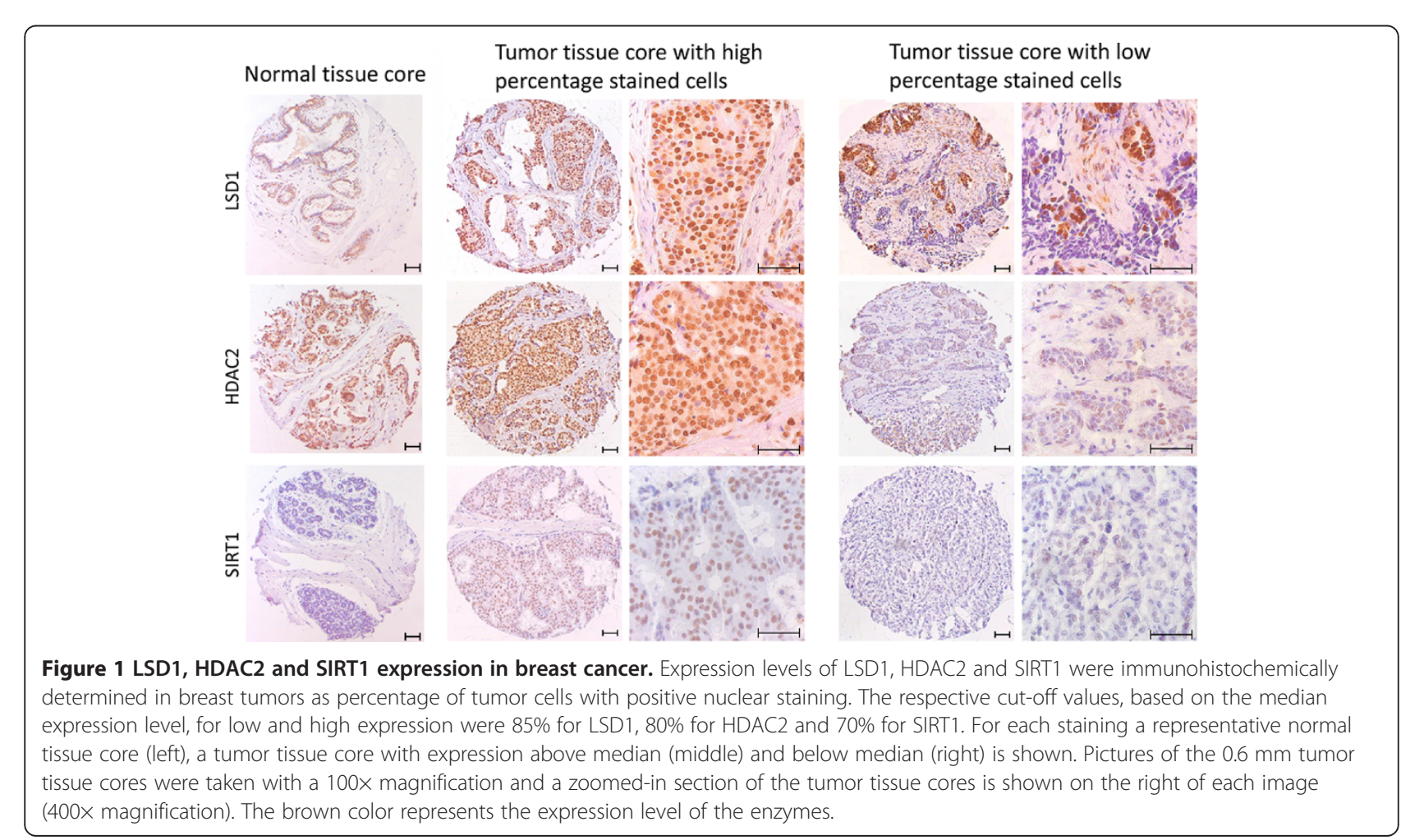


Therefore, a re-evaluation of the scoring was performed by the two observers until agreement was reached. For normal tissues the kappa coefficients were 0.693 for LSD1, 0.628 for HDAC2 and 0.605 for SIRT1, which were all considered as substantial agreement as well. The mean percentage of positive nuclei in the cores determined for each patient by the first observer, was used for survival analyses. Figure 2 shows the expression levels of LSD1, HDAC2 and SIRT1 in normal breast tissues compared to tumor tissues. Analyses of paired tumor and normal tissues showed an increased expression of LSD1 and SIRT1 in tumor tissues compared to normal tissues (both $\mathrm{p}<0.001$ ). HDAC2 expression did not significantly differ in tumor tissues compared to normal tissues $(\mathrm{p}=0.4)$.

\section{Correlation of LSD1, HDAC2 and SIRT1 expression in tumor tissue with tumor stage}

To investigate whether expression of each of the histonemodifying enzymes was related to the TNM tumor stage, the mean percentage of positive tumor nuclei was plotted against tumor stage. Figure 3 shows the percentage of positive nuclei in each tumor stage (I-III) for LSD1, HDAC2 and SIRT1. A one-way ANOVA analysis showed significant differences between the tumor stages for LSD1 $(\mathrm{p}<0.001)$ and SIRT1 $(\mathrm{p}=0.04)$ (Figures 3A and 3C). With higher expression in patients diagnosed with a higher tumor stage. HDAC2 did not show a significant difference between the tumor stages $(\mathrm{p}=0.4)$ (Figure 3B).

\section{Prognostic value of single markers}

Univariate analyses showed significant differences in patient survival and tumor relapse between patients with high and low nuclear expression of LSD1 (OS: $\mathrm{p}=0.002, \mathrm{HR}=1.42$, 95\% CI $=1.13-1.77$; RFS: $\mathrm{p}=0.001, \mathrm{HR}=1.55,95 \% \mathrm{CI}=$ 1.20-1.99) and SIRT1 (RFS: $\mathrm{p}=0.03, \mathrm{HR}=1.32,95 \% \mathrm{CI}=$ 1.03-1.70) (Figures 4A, 4C and 4D). No significant differences were observed for HDAC2 expression (OS: $\mathrm{p}=0.1$, $\mathrm{HR}=1.23,95 \% \mathrm{CI}=0.99-1.54 ; \mathrm{RFS}: \mathrm{p}=0.1, \mathrm{HR}=1.25$, 95\% CI $=0.98-1.61$ ) (Figure 4B and 4D). Multivariate analyses of the expression levels for individual markers showed a significant difference in RFS for SIRT1 ( $p=0.02, H R=$
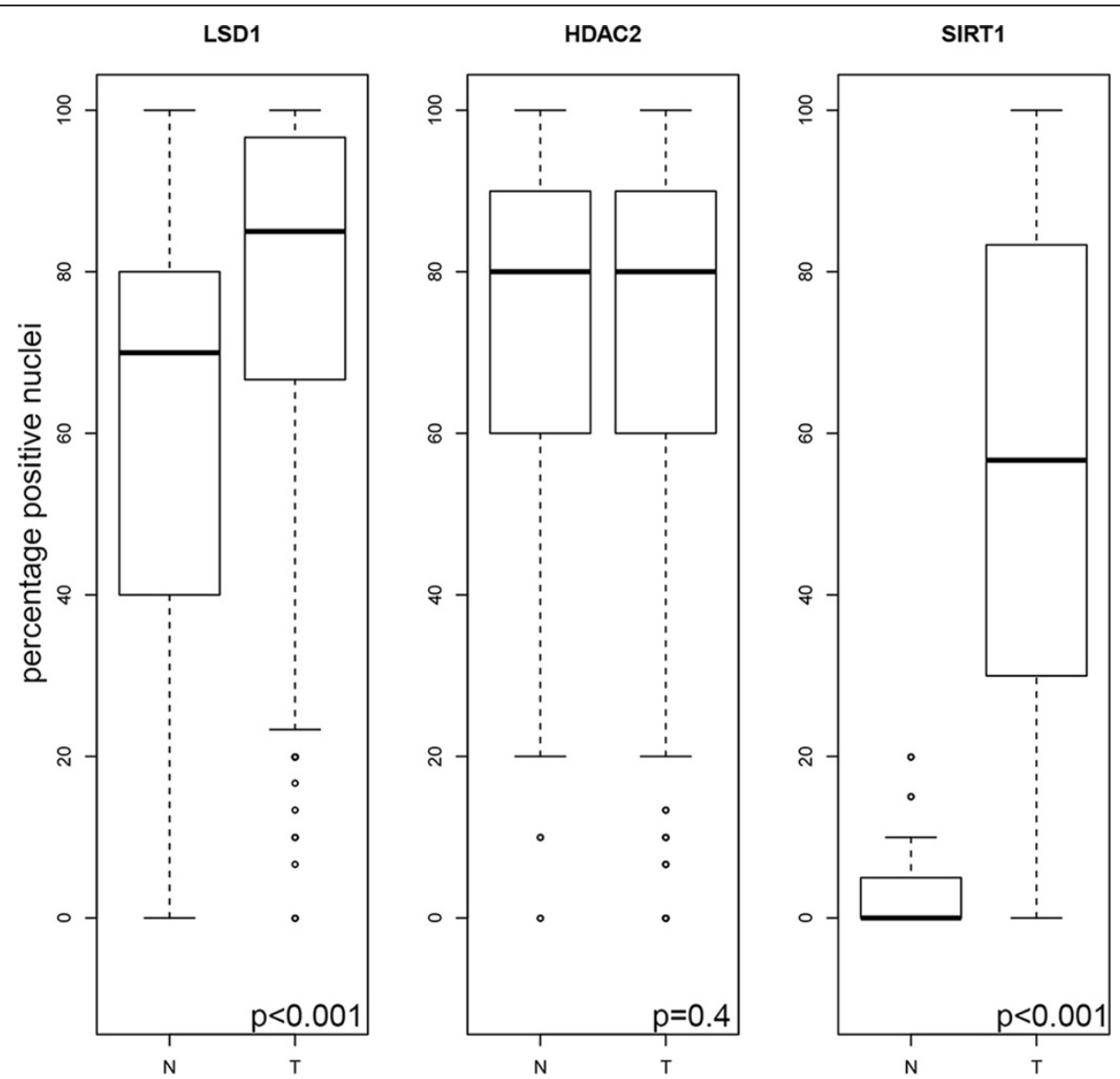

Figure 2 SIRT1, HDAC2 and LSD1 expression in breast tumor tissues compared with normal epithelial breast tissues. The boxplots show the mean percentage (horizontal line) of nuclei positive for LSD1, HDAC2 and SIRT1 in normal epithelial breast cells (labeled "N") versus tumor breast cells (labeled "T") for 60 patients with expression data of the histone-modifying enzymes for tumor tissues and normal epithelial tissues. Outliers are represented by circles. P-values were calculated using a paired student's t-test and p-values $\leq 0.05$ are considered as significant. 

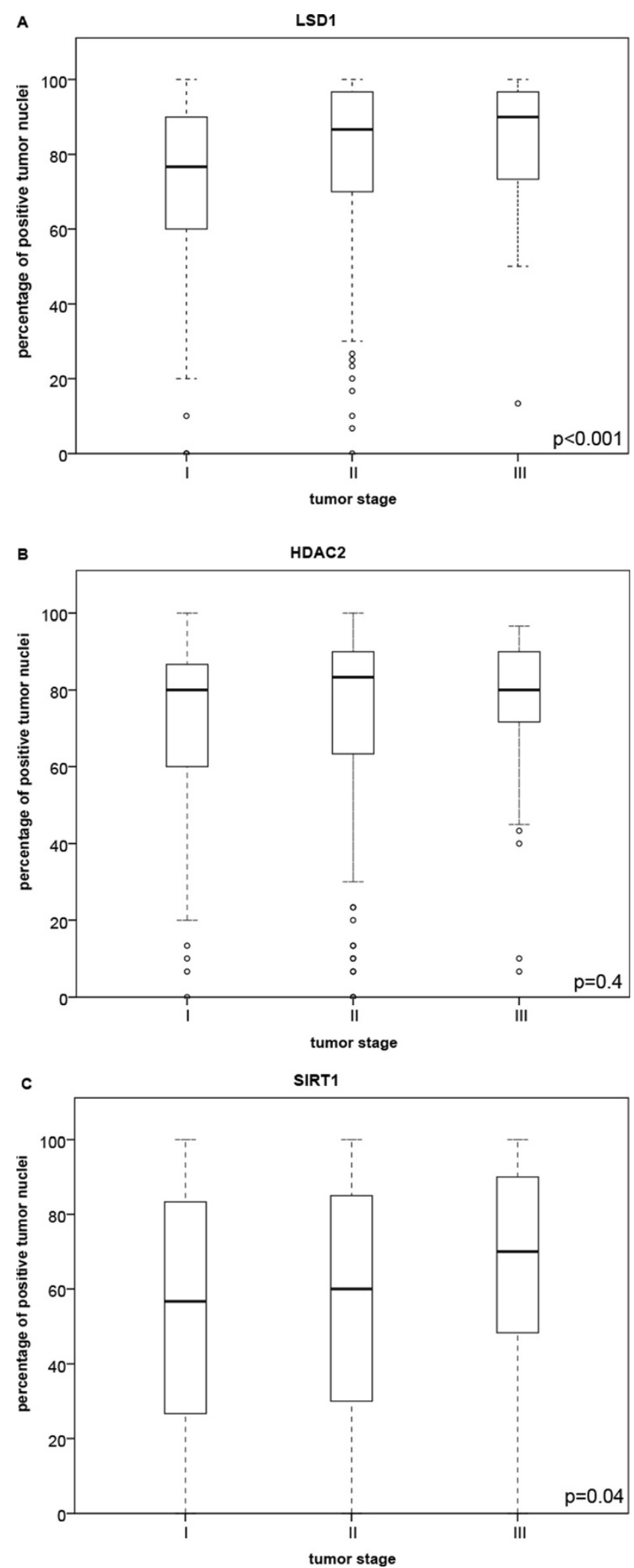

Figure $\mathbf{3}$ (See legend on next page.) 
(See figure on previous page.)

Figure 3 Expression of LSD1, HDAC2 and SIRT1 in different tumor stages. We included 182 patients with a stage I disease, 227 patients with a stage II disease and 51 patients with a stage III tumor. (A) Boxplot showing the percentage of positive tumor cells for LSD1 versus the TNM tumor stage (I-III) at moment of diagnosis. (B) HDAC2 expression levels versus TNM tumor stage shown in a boxplot. (C) The expression levels of SIRT1 versus TNM tumor stage represented in a boxplot. The thick horizontal lines represent the mean percentage of positive tumor cells in each category. Outliers are represented by circles. The $p$-values were calculated using the one-way ANOVA method and $p$-values $\leq 0.05$ are considered as significant.

$1.34,95 \% \mathrm{CI}=1.04-1.74)$ with shorter RFS in the high expression group (Figure 4D). No significant differences were observed for HDAC2 (OS: $\mathrm{p}=0.6, \mathrm{HR}=1.07,95 \% \mathrm{CI}=$ 0.85-1.34; RFS: $\mathrm{p}=0.2, \mathrm{HR}=1.16,95 \% \mathrm{CI}=0.90-1.50)$ and LSD1 (OS: $\mathrm{p}=0.2, \mathrm{HR}=1.18,95 \% \mathrm{CI}=0.94-1.50$; RFS: $\mathrm{p}=$ $0.1, \mathrm{HR}=1.23,95 \% \mathrm{CI}=0.94-1.60)$ in the multivariate analyses (Figure 4D).

\section{Prognostic value of the markers combined}

Since the three enzymes work together in one complex, we hypothesized that the combined expression levels of the three histone-modifying enzymes is a stronger predictor for patient survival and tumor relapse than expression of individual enzymes. Survival analyses of OS and RFS showed that the combined expression level of LSD1, HDAC2 and SIRT1 in breast tumors was more predictive for patient survival and tumor relapse than each of the individual markers separately in both univariate and multivariate analyses (Figure 5). Chi-square analyses showed that there were significant differences between the four patient groups in ER ( $p=0.019)$, PgR $(\mathrm{p}=0.007)$, tumor grade $(\mathrm{p}<0.001)$ and systemic therapy
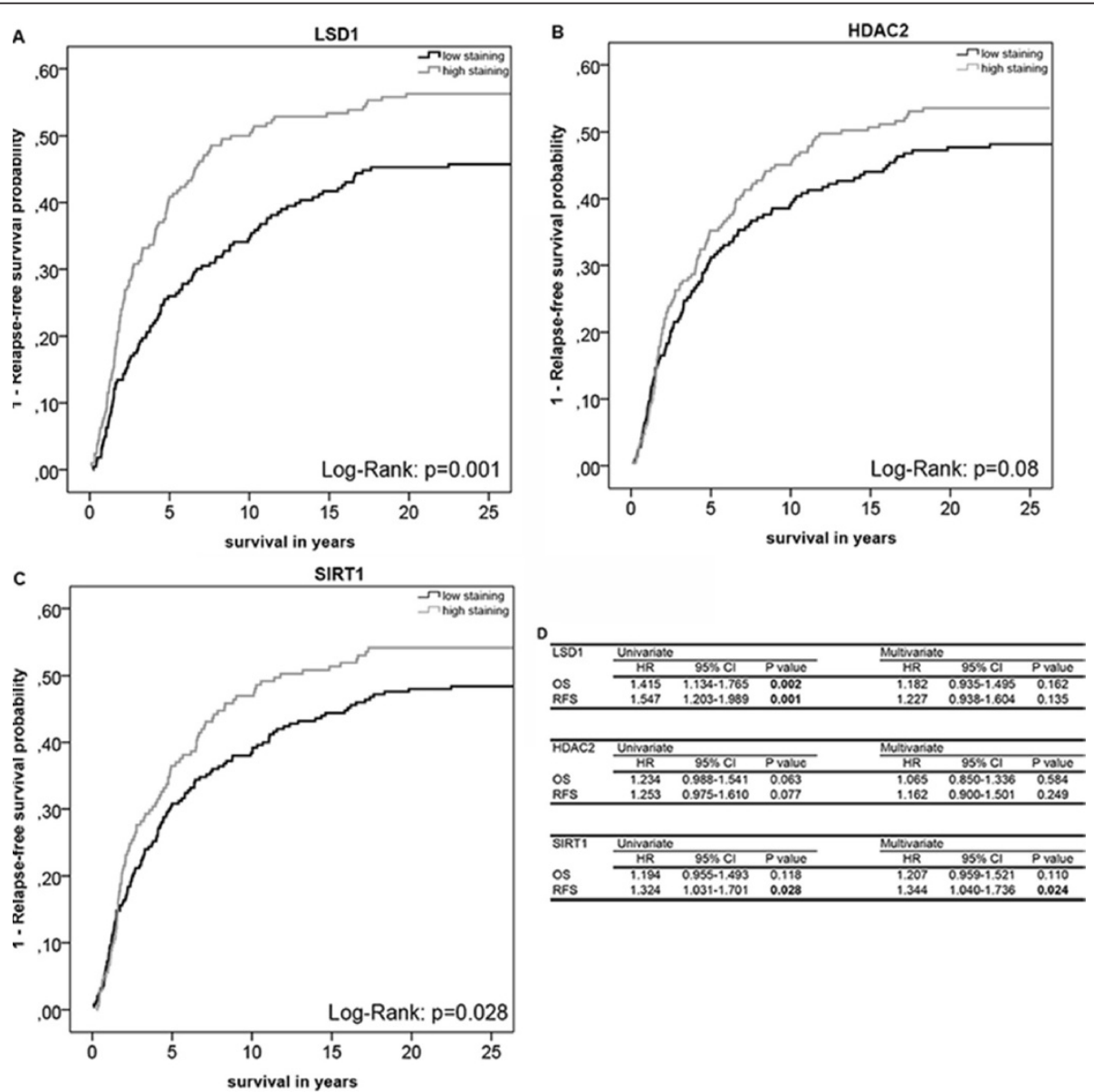

Figure 4 Overall and relapse-free survival analyses of the expression levels LSD1, HDAC2 and SIRT1. Cumulative incidence curves of the univariate relapse-free survival (RFS) analysis of LSD1 (A), HDAC2 (B), and SIRT1 (C) in breast tumors ( $n=460)$. 'Low expression' was defined as expression level below median or equal to median and 'high expression' was defined as expression level above median. (D) Hazard ratios (HR), their $95 \%$ confidence intervals $(95 \% \mathrm{Cl}$ ) and their corresponding p-values for LSD1, HDAC2, and SIRT1 expression for overall survival (OS) and RFS were evaluated with the Cox proportional hazard model for uni- and multivariate analysis. Significant $p$-values $(\leq 0.05)$ are indicated in bold. 


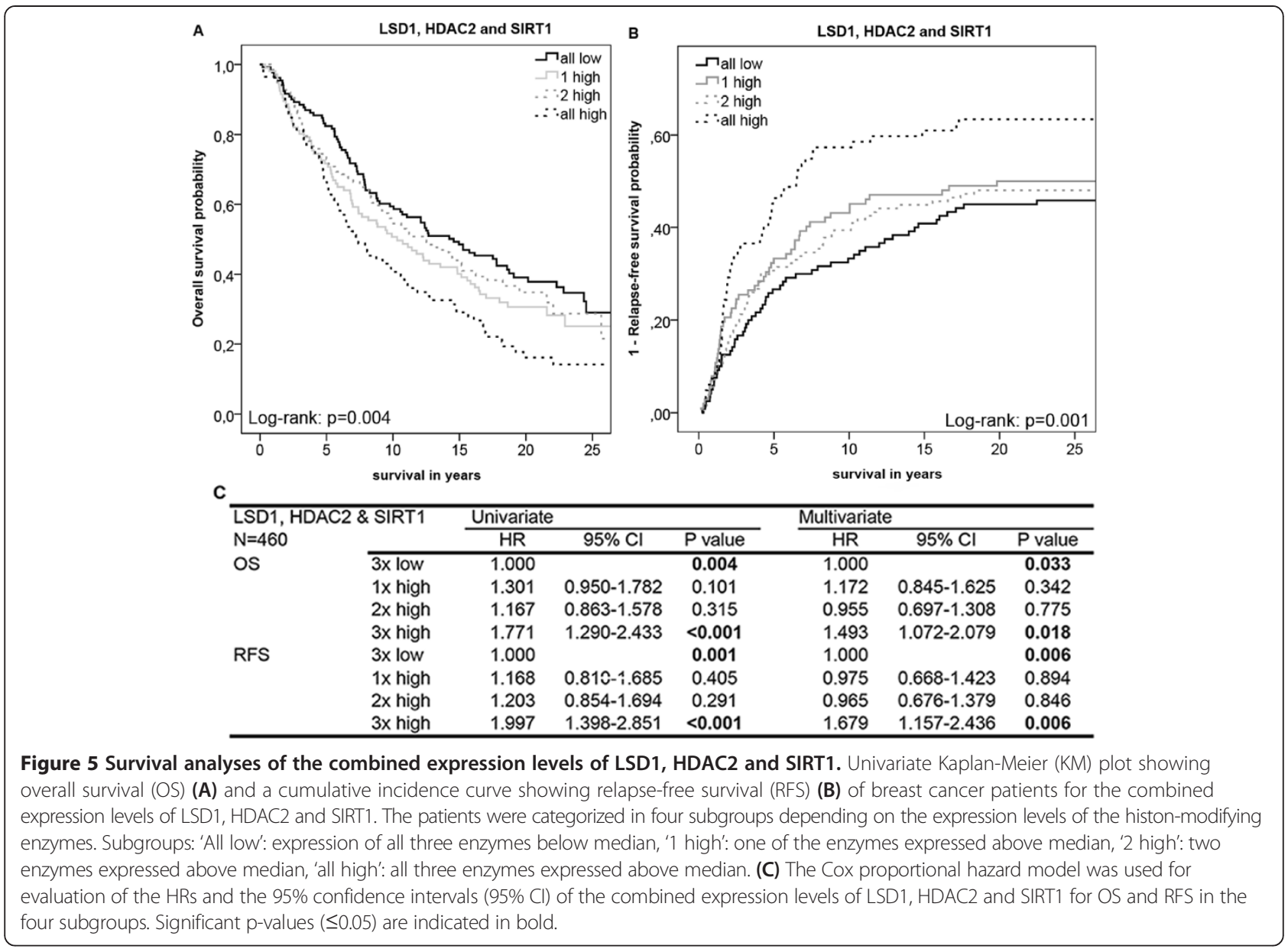

( $\mathrm{p}=0.010$ ), for which we corrected in the multivariate analyses. Multivariate analyses of the combined marker expression levels showed that patients with high expression level of all three markers had a shorter OS compared to patients with low expression of all the enzymes ( $\mathrm{p}=$ $0.03, \mathrm{HR}=1.49,95 \% \mathrm{CI}=1.07-2.08$ ) (Figure $5 \mathrm{C}$ ). For RFS the $\mathrm{HR}$ was $1.68(\mathrm{p}=0.006,95 \% \mathrm{CI}=1.16-2.44)$ in the 'all-high' expression group versus the 'all-low' expression group (Figure 5C). This result indicated that patients with high expression of all three enzymes have a shorter RFS compared to patients with one or more enzymes with a low expression level.

\section{Correlation of the combined expression levels of LSD1, HDAC2 and SIRT1 with tumor differentiation and tumor cell proliferation}

We tested if there was a correlation between the combined expression levels of the three enzymes and tumor differentiation, a marker of aggressive tumors, in the whole study population. Indeed, a significant correlation between these expression levels and tumor differentiation was found ( $\mathrm{p}<0.001$; Table 2$)$. The results showed that $24 \%$ of the patients with low expression of all three enzymes had a well-differentiated tumor and only $12 \%$ of the patients with high expression of all three enzymes had a well-differentiated tumor. A low differentiation grade was found in $21 \%$ of patients with low expression of LSD1, HDAC2 and SIRT1 and 43\% of the patients with high expression of all three enzymes had a low grade of tumor differentiation. In addition, we investigated the relation between the combined expression levels of LSD1, HDAC2 and SIRT1 and tumor cell proliferation, assessed by ki-67 expression, which is another marker of aggressive tumors. Ki-67 expression levels were determined by IHC previously in our study cohort [21] and data were available for 423 of 460 patients (92\%). A significant correlation was found between the expression of ki-67 and the combined expression levels of the three enzymes $(p=0.002$; Table 3$)$. The results showed that in $68 \%$ of the patients with low expression of all three enzymes, there was no expression of ki-67, which indicated that there is only a low level of tumor cell proliferation in these patients. When at least one of the three histone-modifying enzymes showed high expression, we observed an increase in the percentage of ki-67 positive tumors (up to 56\%), indicating more 
Table 2 Correlation between combined expression level of LSD1, HDAC2 and SIRT1 and tumor differentiation

\begin{tabular}{|c|c|c|c|c|c|}
\hline $\begin{array}{l}\mathrm{N}=460 \\
\text { Tumor diff }\end{array}$ & $3 \times$ low & $1 \times$ high & $2 \times$ high & $3 \times$ high & Total \\
\hline High & $31(23.7 \%)$ & $9(8.4 \%)$ & $21(15.4 \%)$ & $10(11.6 \%)$ & 71 (15.4\%) \\
\hline Moderate & 73 (55.7\%) & 52 (49.1\%) & 58 (42.3\%) & $39(45.4 \%)$ & $222(48.3 \%)$ \\
\hline Low & $27(20.6 \%)$ & $45(42.5 \%)$ & $58(42.3 \%)$ & $37(43.0 \%)$ & 167 (36.3\%) \\
\hline Total & 131 & 106 & 137 & 86 & 460 \\
\hline
\end{tabular}

Tumor differentiation, according to tumor grade as assessed by an experienced pathologist, versus the combined expression levels of LSD1, HDAC2 and SIRT1 in 460 breast cancer patients are shown. Patients were divided in four subgroups based on the expression levels of the histone-modifying enzymes: all enzymes below median ( $3 x$ low), one enzyme above median ( $1 \times$ high), two enzymes above median ( $2 \times$ high) and all three enzymes above median ( $3 x$ high).

proliferation of the tumor cells in these patients. In summary, there are correlations between the combined expression levels of LSD1, HDAC2 and SIRT1 and tumor differentiation and between the combined expression levels of these enzymes and tumor cell proliferation.

\section{Discussion}

Our study identified combined expression levels of the histone-modifying enzymes LSD1, HDAC2 and SIRT1 as an independent prognostic factor for patient survival and tumor relapse in breast cancer patients. In addition, our results showed that the combined marker expression levels correlated with tumor differentiation and tumor cell proliferation. All these results implicated that high expression of all three enzymes is associated with a more aggressive phenotype of the breast tumors.

Histone-modifying enzymes are involved in numerous processes that are related to cancer, including cellular proliferation and differentiation [22]. There is increasing evidence that shows that aberrant expression of these enzymes has a role in (breast) cancer development and tumor growth $[5,6,8,9,23]$. LSD1 is overexpressed in various cancer types, such as bladder, lung and colorectal cancer [23]. In our breast cancer patient study cohort, an increase in the expression of LSD1 in tumor tissues was found compared with normal epithelial breast tissues. Our study also showed an increase in nuclear expression of LSD1 from tumor stage I to III, which has been described in literature by another group as well [5].
Furthermore, we demonstrated that SIRT1 expression levels were significantly increased in tumor tissues compared to normal epithelial breast tissues, which has also been described in literature [9]. The multivariate Cox proportional hazard analyses showed that SIRT1 expression was an independent prognostic factor for RFS, but not for OS in our breast cancer cohort, although a previous publication showed prognostic value for both [10]. This discrepancy can be explained by differences between patient cohorts, because our cohort contained older patients and we excluded patients with a TNM tumor stage IV disease from the study. In our cohort, HDAC2 expression was not significantly different in normal and tumor breast tissues and was not predictive for OS and RFS, confirming the results of the univariate OS analysis of Müller et al. [24].

Other groups have studied combinations of histonemodifying enzymes, but did not correlate these to clinical outcome. For example, Huang et al. showed in vitro that LSD1 and HDACs are involved in tumor cell proliferation, because synergistic inhibition of breast cancer cell proliferation was observed as compared to inhibition of the individual enzymes [11]. In the same study, microarray screening showed that inhibition of the enzymes led to reexpression of aberrantly silenced genes involved in processes such as cell differentiation and cell proliferation, which are frequently deregulated in breast cancer [11].

Our study is, to our knowledge, the first study that correlated the combined nuclear expression levels of these

Table 3 Correlation between combined expression level of LSD1, HDAC2 and SIRT1 and ki-67 expression

\begin{tabular}{llllll}
\hline $\mathbf{N}=\mathbf{4 2 3}$ & $\mathbf{3 \times}$ low & $\mathbf{1 \times}$ high & $\mathbf{2 \times}$ high & $\mathbf{3 \times}$ high & \\
Ki-67 & & & & total \\
\hline No expression & $81(67.5 \%)$ & $40(44.0 \%)$ & $67(51.1 \%)$ & $37(45.7 \%)$ & $225(53.2 \%)$ \\
Expression & $39(32.5 \%)$ & $51(56.0 \%)$ & $64(48.9 \%)$ & $44(54.3 \%)$ & $198(46.8 \%)$ \\
Total & 120 & 91 & 131 & 81 & 423
\end{tabular}

Chi-square: $p=0.002$

Ki-67 expression versus the combined expression levels of LSD1, HDAC2 and SIRT1 in 423 breast cancer patients are shown. Patients were divided into four categories based on the expression levels of the histone-modifying enzymes: all enzymes below median ( $3 x$ low), one enzyme above median ( $1 \times$ high), two enzymes above median ( $2 \times$ high) and all three enzymes above median ( $3 \times$ high). 
three histone-modifying enzymes with survival data in breast cancer patients. High expression of all three enzymes in tumor cells was correlated with reduced patient survival and shortened RFS compared to the expression level of the individual enzymes, implicating that LSD1, HDAC2 and SIRT1 act together in the same complex.

It has been shown in literature that all three histonemodifying enzymes, analyzed in our study, are individually involved in inhibition of functioning of p53 via direct modification of p53 (demethylation by LSD1 [25] and deacetylation by SIRT1 [26]) or inhibition of p53-DNA binding (HDAC2 [27]). p53 is a well-known tumor-suppressor and reduced functioning of p53 leads to reduced apoptosis, reduced cellular senescence and increased survival of cells with DNA-damage, due to reduced cell-cycle arrests, potentially leading to tumor development [25-27]. Therefore, we hypothesize that the complex of LSD1, HDAC2 and SIRT1 has important roles, next to chromatin repression, in regulating cell survival and that aberrant expression of this complex leads to sustained survival of tumor cells. Possibly, combined inhibition of multiple histone-modifying enzymes, such as LSD1, HDAC2 and SIRT1, could lead to improved treatment of breast cancer patients.

\section{Conclusions}

In summary, we showed that the combined expression level of LSD1, HDAC2 and SIRT1 is a good predictor for OS and RFS in breast cancer patients. High expression of all three enzymes correlated with a more aggressive tumor phenotype, which makes this multi-enzyme complex an interesting target for breast cancer treatment. Future research for prognostic biomarkers should focus on analyses of such combinations of histone-modifying enzymes, acting together in multi-protein complexes, and their respective histone modifications. This can potentially further elucidate the complex epigenetic regulatory mechanisms in breast cancer, which will help identifying new targets for therapy.

\footnotetext{
Abbreviations

BCS: Breast conserving surgery; Cl: Confidence interval; ER: Estrogen receptor; FFPE: Formalin-fixed paraffin-embedded; H3K4: Lysine 4 on histone 3; H3K9: Lysine 9 on histone 3; HDAC2: Histone deacetylase 2; HER2: Human epidermal growth factor 2; HR: Hazard ratio; IHC: Immunohistochemistry; KM: Kaplan-Meier; LSD1: Lysine-specific demethylase 1; OS: Overall survival; PgR: Progesterone receptor; RFS: Relapse-free survival; RT: Radiotherapy; SIRT1: Silent mating-type information regulation 2 homologue 1; TMA: Tissue microarray.
}

\section{Competing interests}

The authors declare that they have no competing interests.

\section{Authors' contributions}

RSD and $A Q v H$ contributed equally to this study. AQvH, AB, CJHvdV and PJKK designed and coordinated the study. EdK was responsible for creation of the TMA and composed the clinical database. AQvH set up and performed the immunohistochemistry. VTHBMS was the pathologist who graded and histologically classified all tumors. NGDE and AS were involved in scoring of the immunohistochemistry staining. RSD, IJGB and EB were involved in the statistical analyses. RSD and PJKK drafted the manuscript. $A Q v H, A B, I J G B, E d K, E B$ and $C J H v d V$ reviewed and edited the manuscript. All authors have read and approved the final manuscript.

\section{Acknowledgements}

We thank Dilesh Kishoendajal for his efforts to optimize the antibodies used for the immunohistochemistry. We thank Gerrit-Jan Liefers (LUMC) for his clinical input in the study. We would like to thank the Dutch Cancer Society (KWF 2007-3968) for funding part of the research performed in this study.

\section{Author details}

'Department of Surgery, K6-R, Leiden University Medical Center (LUMC), P.O. Box 9600, 2300 RC Leiden, The Netherlands. ${ }^{2}$ Department of Pathology, LUMC, Leiden, The Netherlands.

Received: 4 March 2014 Accepted: 8 August 2014

Published: 20 August 2014

\section{References}

1. Goldhirsch A, Wood WC, Gelber RD, Coates AS, Thurlimann B, Senn HJ: Progress and promise: highlights of the international expert consensus on the primary therapy of early breast cancer 2007. Ann Oncol 2007, 18:1133-1144

2. Marme F, Schneeweiss A: Personalized therapy in breast cancer. Onkologie 2012, 35(Suppl 1):28-33.

3. Nowsheen S, Aziz K, Tran PT, Gorgoulis VG, Yang ES, Georgakilas AG: Epigenetic inactivation of DNA repair in breast cancer. Cancer Lett 2012, 342:213-222.

4. Klose RJ, Zhang Y: Regulation of histone methylation by demethylimination and demethylation. Nat Rev Mol Cell Biol 2007, 8:307-318.

5. Serce N, Gnatzy A, Steiner S, Lorenzen H, Kirfel J, Buettner R: Elevated expression of LSD1 (Lysine-specific demethylase 1) during tumour progression from pre-invasive to invasive ductal carcinoma of the breast. BMC Clin Pathol 2012, 12:13.

6. Glozak MA, Seto E: Histone deacetylases and cancer. Oncogene 2007, 26:5420-5432.

7. Munster PN, Thurn KT, Thomas S, Raha P, Lacevic M, Miller A, Melisko M, Ismail-Khan R, Rugo H, Moasser M, Minton SE: A phase II study of the histone deacetylase inhibitor vorinostat combined with tamoxifen for the treatment of patients with hormone therapy-resistant breast cancer. Br J Cancer 2011, 104:1828-1835.

8. Liu T, Liu PY, Marshall GM: The critical role of the class III histone deacetylase SIRT1 in cancer. Cancer Res 2009, 69:1702-1705.

9. Sung JY, Kim R, Kim JE, Lee J: Balance between SIRT1 and DBC1 expression is lost in breast cancer. Cancer Sci 2010, 101:1738-1744.

10. Lee H, Kim KR, Noh SJ, Park HS, Kwon KS, Park BH, Jung SH, Youn HJ, Lee BK, Chung MJ, Koh DH, Moon WS, Jang KY: Expression of DBC1 and SIRT1 is associated with poor prognosis for breast carcinoma. Hum Pathol 2011, 42:204-213.

11. Huang Y, Vasilatos SN, Boric L, Shaw PG, Davidson NE: Inhibitors of histone demethylation and histone deacetylation cooperate in regulating gene expression and inhibiting growth in human breast cancer cells. Breast Cancer Res Treat 2012, 131:777-789.

12. Vasilatos SN, Katz TA, Oesterreich S, Wan Y, Davidson NE, Huang Y: Crosstalk between lysine-specific demethylase 1 (LSD1) and histone deacetylases mediates antineoplastic efficacy of HDAC inhibitors in human breast cancer cells. Carcinogenesis 2013, 34:1196-1207.

13. Mulligan P, Yang F, Di SL, Ji JY, Ouyang J, Nishikawa JL, Toiber D, Kulkarni M, Wang Q, Najafi-Shoushtari SH, Mostoslavsky R, Gygi SP, Gill G, Dyson NJ, Naar AM: A SIRT1-LSD1 corepressor complex regulates Notch target gene expression and development. Mol Cell 2011, 42:689-699.

14. de Kruijf EM, Engels CC, van de Water W, Bastiaannet E, Smit VT, van de Velde $C J$, Liefers GJ, Kuppen PJ: Tumor immune subtypes distinguish tumor subclasses with clinical implications in breast cancer patients. Breast Cancer Res Treat 2013, 142:355-364.

15. Federation of Medical Scientific Societies: Code for Proper Secondary Use of Human Tissue in the Netherlands. 43. http://www.federa.org/sites/ default/files/bijlagen/coreon/codepropersecondaryuseofhumantissue1_0.pdf. 
16. McShane LM, Altman DG, Sauerbrei W, Taube SE, Gion M, Clark GM: REporting recommendations for tumour MARKer prognostic studies (REMARK). Br J Cancer 2005, 93:387-391.

17. Gu H, Roizman B: Engagement of the lysine-specific demethylase/ HDAC1/CoREST/REST complex by herpes simplex virus 1. J Virol 2009, 83:4376-4385.

18. Lin Y, Wu Y, Li J, Dong C, Ye X, Chi YI, Evers BM, Zhou BP: The SNAG domain of Snail 1 functions as a molecular hook for recruiting lysine-specific demethylase 1. EMBO J 2010, 29:1803-1816.

19. Zhang Y, Zhang M, Dong H, Yong S, Li X, Olashaw N, Kruk PA, Cheng JC Bai W, Chen J, Nicosia SV, Zhang X: Deacetylation of cortactin by SIRT1 promotes cell migration. Oncogene 2009, 28:445-460.

20. van Nes JG, de Kruijf EM, Faratian D, van de Velde CJ, Putter H, Falconer C, Smit VT, Kay C, van de Vijver MJ, Kuppen PJ, Bartlett JM: COX2 expression in prognosis and in prediction to endocrine therapy in early breast cancer patients. Breast Cancer Res Treat 2011, 125:671-685.

21. Engels CC, Ruberta F, de Kruijf EM, van Pelt GW, Smit VT, Liefers GJ, Matsushima T, Shibayama M, Ishihara H, van de Velde CJ, Kuppen PJ: The prognostic value of apoptotic and proliferative markers in breast cancer. Breast Cancer Res Treat 2013, 142:323-339.

22. Hanahan D, Weinberg RA: Hallmarks of cancer: the next generation. Cell 2011, 144:646-674

23. Hayami S, Kelly JD, Cho HS, Yoshimatsu M, Unoki M, Tsunoda T, Field HI, Neal DE, Yamaue $H$, Ponder BA, Nakamura $Y$, Hamamoto R: Overexpression of LSD1 contributes to human carcinogenesis through chromatin regulation in various cancers. Int J Cancer 2011, 128:574-586.

24. Muller BM, Jana L, Kasajima A, Lehmann A, Prinzler J, Budczies J, Winzer KJ, Dietel $M$, Weichert W, Denckert C: Differential expression of histone deacetylases HDAC1, 2 and 3 in human breast cancer-overexpression of HDAC2 and HDAC3 is associated with clinicopathological indicators of disease progression. BMC Cancer 2013, 13:215.

25. Huang J, Sengupta R, Espejo AB, Lee MG, Dorsey JA, Richter M, Opravil S, Shiekattar R, Bedford MT, Jenuwein T, Berger SL: p53 is regulated by the lysine demethylase LSD1. Nature 2007, 449:105-108.

26. Chen WY, Wang DH, Yen RC, Luo J, Gu W, Baylin SB: Tumor suppressor HIC1 directly regulates SIRT1 to modulate p53-dependent DNA-damage responses. Cell 2005, 123:437-448.

27. Harms KL, Chen X: Histone deacetylase 2 modulates $p 53$ transcriptional activities through regulation of p53-DNA binding activity. Cancer Res 2007, 67:3145-3152.

doi:10.1186/1471-2407-14-604

Cite this article as: Derr et al:: High nuclear expression levels of histonemodifying enzymes LSD1, HDAC2 and SIRT1 in tumor cells correlate with decreased survival and increased relapse in breast cancer patients. BMC Cancer 2014 14:604.

\section{Submit your next manuscript to BioMed Central and take full advantage of:}

- Convenient online submission

- Thorough peer review

- No space constraints or color figure charges

- Immediate publication on acceptance

- Inclusion in PubMed, CAS, Scopus and Google Scholar

- Research which is freely available for redistribution 